\title{
Behaviour of augmented Lagrangian and Hamiltonian methods for multibody dynamics in the proximity of singular configurations
}

\author{
Francisco González, Daniel Dopico, Roland Pastorino, Javier Cuadrado
}

This is a post-peer-review, pre-copyedit version of an article published in Nonlinear Dynamics. The final authenticated version is available online at:

http://dx.doi.org/10.1007/s11071-016-2774-5.

\begin{abstract}
Augmented Lagrangian methods represent an efficient way to carry out the forwarddynamics simulation of mechanical systems. These algorithms introduce the constraint forces in the dynamic equations of the system through a set of multipliers. While most of these formalisms were obtained using Lagrange's equations as starting point, a number of them have been derived from Hamilton's canonical equations. Besides being efficient, they are generally considered to be robust, which makes them especially suitable for the simulation of systems with discontinuities and impacts. In this work, we have focused on the simulation of mechanical assemblies that undergo singular configurations. First, some sources of numerical difficulties in the proximity of singular configurations were identified and discussed. Afterwards, several augmented Lagrangian and Hamiltonian formulations were compared in terms of their robustness during the forward-dynamics simulation of two benchmark problems. Newton-Raphson iterative schemes were developed for these formulations with the Newmark formula as numerical integrator. These outperformed fixed point iteration approaches in terms of robustness and efficiency. The effect of the formulation parameters on simulation performance was also assessed.
\end{abstract}

Keywords: Multibody system dynamics, augmented Lagrangian methods, Hamiltonian methods, singular configurations 


\section{Introduction}

Forward-dynamics simulation of multibody systems is a relatively new area in the field of Mechanics. The progress in computer architectures and software tools during the last decades has boosted both research and industry applications of this technique. At the same time, the expectations regarding the capabilities and performance of multibody codes have also increased. Among the many applications of multibody dynamics, real-time environments such as Humanand Hardware-in-the-Loop (HiL) setups are especially demanding in terms of both efficiency and robustness. As a consequence, a considerable effort has been made within the multibody community to develop fast and reliable simulation algorithms to satisfy these requirements.

Generally speaking, multibody systems consist of a set of rigid or flexible links interconnected by joints. The consideration of the kinematic constraints introduced by the latter usually leads to the need for expressing the dynamics equations as a system of Differential Algebraic Equations (DAEs). Many approaches can be used to deal with these equations, among which Lagrange's multiplier method is a widely used one [16].

If a mechanical system is described with a set of $n$ generalized coordinates $\mathbf{q}$, subjected to $m$ holonomic kinematic constraints $\boldsymbol{\Phi}$, the equations of motion can be expressed as

$$
\begin{aligned}
\mathbf{M} \ddot{\mathbf{q}}+\mathbf{c} & =\mathbf{f}+\mathbf{f}_{c} \\
\mathbf{\Phi}(\mathbf{q}, t) & =\mathbf{0}
\end{aligned}
$$

where $\mathbf{M}$ is the $n \times n$ mass matrix, c contains the Coriolis and centrifugal forces, and $\mathbf{f}$ and $\mathbf{f}_{c}$ are the applied and constraint forces, respectively. Following a Lagrangian approach, the generalized constraint reactions can be expressed as $\mathbf{f}_{c}=-\boldsymbol{\Phi}_{\mathbf{q}}^{\mathrm{T}} \boldsymbol{\lambda}$, where $\boldsymbol{\Phi}_{\mathbf{q}}=\partial \boldsymbol{\Phi} / \partial \mathbf{q}$ is the $m \times n$ Jacobian matrix of the constraints and $\boldsymbol{\lambda}$ is a set of $m$ Lagrange multipliers.

One of the first augmented Lagrangian algorithms for multibody dynamics was introduced by Bayo et al. [4]. The proposed method combined a penalty representation of the constraint forces with an iterative update of the Lagrange multipliers. An extension of the method to handle nonholonomic constraints was also included in [4]. Several related formalisms based on the augmented Lagrangian approach have been subsequently developed. An implementation of the algorithm in [4] aiming at real-time efficiency was published in [3]. In [6] and [11] mass-orthogonal projections were used together with the augmented-Lagrangian formulation to 
ensure the satisfaction of the kinematic constraints. These two papers included index- 3 versions of the algorithms as well, in which the dynamic equations were combined with the numerical integrator formulas. The resulting system of DAEs was solved iteratively following a NewtonRaphson scheme, thus improving the robustness of the method. The original algorithms in [6] and [11] were designed for holonomic constraints alone; an index-3 augmented Lagrangian algorithm able to deal with nonholonomic constraints was later described in [12]. The above mentioned formalisms and similar ones, e.g. [25], have been successfully used in the study and simulation of a wide variety of mechanical systems. Application examples include heavy machinery simulators [13], biomechanics [29], and co-simulation settings for vehicle dynamics [18].

It is also possible to obtain the dynamics equations using Hamilton's canonical equations as starting point. Following this approach, the unconstrained equations of motion become a system of $2 n$ first order Ordinary Differential Equations (ODEs), instead of the system of $n$ second order ODEs in Eq. (1a). Augmented Lagrangian algorithms based on Hamilton's canonical equations can also be found in the field of multibody dynamics, e.g. [5], [2]. It was stated in [2] that the methods based on canonical equations are more robust than their classical augmented Lagrangian counterparts and ensure a better satisfaction of the kinematic constraints. This was supported by the performance comparison of two formulations, one representative of each approach, in the dynamic simulation of mechanical systems with singular configurations. Although both algorithms were able to deal with the test problems, the Hamiltonian one did not show pathological behaviour in any of the simulations carried out by the authors. However, these formulations have received comparatively less attention in the literature since they were first presented to the multibody community. Naudet et al. [27] developed a recursive algorithm based on canonical momenta, although they did not follow an augmented Lagrangian approach. The authors affirm in [27] that a possible reason why Hamiltonian equations are rather infrequent in multibody applications is that they are computationally intensive to construct and they cannot compete with acceleration-based algorithms, especially recursive ones. More recently, Malczyk et al. [26] combined the Divide and Conquer Algorithm (DCA) [14] with Hamilton's canonical equations to obtain a parallel algorithm. Their preliminary results suggested that their Hamiltonian approach can outperform the Lagrangian one in terms of accuracy in the enforcement of kinematic constraints and conservation of the mechanical energy of the system.

In the research reported in this paper, the behaviour of augmented Lagrangian and Hamilto- 
nian methods for multibody dynamics was studied in the proximity of singular configurations. It was shown that even though these formalisms are able to deal with redundant kinematic constraints and rank deficient Jacobian matrices, they still suffer from numerical difficulties when they are employed to simulate mechanisms that go through singularities. The cause of these numerical problems was determined to be the introduction of impact loads in the constraint reactions, which in turn was motivated by the enlargement of the subspace of admissible motion at the singular points. The factors that determine the ability of a given algorithm to overcome these difficulties were investigated as well. Existing augmented Lagrangian and Hamiltonian methods for multibody dynamics were combined with Newmark integration formulas to develop time-stepping algorithms with Newton-Raphson iterative schemes. The comparison of the new algorithms and other existing formulations was done using test examples from the IFToMM benchmark problem library [21]. Results showed that the selection of the numerical integration routine and the formulation parameters has a critical effect on the stability and efficiency of the simulation. Newton-Raphson implementations showed a more robust behaviour than their fixed point counterparts and were able to withstand the impact forces introduced by the singularities while avoiding large variations in the mechanical energy of the system.

\section{Augmented Lagrangian formulations}

Several formulations were selected for this study among the many available in the literature. The ones described in [4], [2], and [6] were chosen because they share a similar dynamics equations structure. Newton-Raphson implementations of the original algorithms were also developed and implemented. In the following, natural coordinates [22] are assumed to be used in the modelling. This has two important consequences. First, term $\mathbf{c}$ vanishes from the dynamics equations. Second, with a proper selection of coordinates the mass matrix $\mathbf{M}$ becomes constant, and so all its derivatives are zero.

\subsection{Penalty formulation}

Even though it is not an augmented Lagrangian one, it is convenient to briefly describe here the penalty formulation introduced in [4], as it is the starting point for the development of a large 
set of augmented Lagrangian algorithms. This formulation replaces the kinematic constraints $\boldsymbol{\Phi}=\mathbf{0}$ with penalty mass-spring-damper systems. This is achieved introducing fictitious potential and kinetic energy terms in the integral action $A$ of the mechanical system, as well as a set of dissipative forces. Then, the constraint reactions can be replaced by forces and torques proportional to the constraint violations at the acceleration, velocity, and configuration levels

$$
\boldsymbol{\lambda}=\alpha\left(\ddot{\mathbf{\Phi}}+2 \xi \omega \dot{\boldsymbol{\Phi}}+\omega^{2} \mathbf{\Phi}\right)
$$

where $\alpha$ is the penalty factor, and $\xi$ and $\omega$ have a physical meaning similar to that of Baumgarte's stabilization parameters [1]. Together with the velocity- and acceleration-level expressions of the kinematic constraints

$$
\begin{aligned}
& \dot{\boldsymbol{\Phi}}=\boldsymbol{\Phi}_{\mathbf{q}} \dot{\mathbf{q}}+\boldsymbol{\Phi}_{t}=\mathbf{0} \\
& \ddot{\boldsymbol{\Phi}}=\boldsymbol{\Phi}_{\mathbf{q}} \ddot{\mathbf{q}}+\dot{\boldsymbol{\Phi}}_{\mathbf{q}} \dot{\mathbf{q}}+\dot{\boldsymbol{\Phi}}_{t}=\mathbf{0}
\end{aligned}
$$

where $\boldsymbol{\Phi}_{t}=\partial \boldsymbol{\Phi} / \partial t$, Eq. (2) allows for the transformation of the system of DAEs (1) into a system of $n$ second order ODEs

$$
\left(\mathbf{M}+\boldsymbol{\Phi}_{\mathbf{q}}^{\mathrm{T}} \alpha \boldsymbol{\Phi}_{\mathbf{q}}\right) \ddot{\mathbf{q}}=\mathbf{f}-\boldsymbol{\Phi}_{\mathbf{q}}^{\mathrm{T}} \alpha\left(\dot{\boldsymbol{\Phi}}_{\mathbf{q}} \dot{\mathbf{q}}+\dot{\mathbf{\Phi}}_{t}+2 \xi \omega \dot{\mathbf{\Phi}}+\omega^{2} \boldsymbol{\Phi}\right)
$$

Terms $\alpha, \xi$, and $\omega$ are $n \times n$ matrices in the general case but for simplicity they are treated as scalars in this document.

\subsection{Augmented Lagrangian formulation}

The penalty formulation in Eq. (5) has the disadvantage of being very sensitive to the value of the penalty factor $\alpha$ in terms of convergence. Additionally, a certain violation of constraints is required to develop the necessary reaction forces $\mathbf{f}_{c}$, so a complete fulfilment of the constraints can never be achieved. The augmented Lagrangian formulations proposed in [4] and [6] intended to overcome these limitations. The Lagrange multiplier method was applied to the solution of 
Eq. (5) to obtain the following iterative algorithm

$$
\begin{aligned}
& \left(\mathbf{M}+\boldsymbol{\Phi}_{\mathbf{q}}^{\mathrm{T}} \alpha \boldsymbol{\Phi}_{\mathbf{q}}\right) \ddot{\mathbf{q}}+\boldsymbol{\Phi}_{\mathbf{q}}^{\mathrm{T}} \boldsymbol{\lambda}^{*}=\mathbf{f}-\boldsymbol{\Phi}_{\mathbf{q}}^{\mathrm{T}} \alpha\left(\dot{\boldsymbol{\Phi}}_{\mathbf{q}} \dot{\mathbf{q}}+\dot{\boldsymbol{\Phi}}_{t}+2 \xi \omega \dot{\boldsymbol{\Phi}}+\omega^{2} \boldsymbol{\Phi}\right) \\
& \boldsymbol{\lambda}_{i+1}^{*}=\boldsymbol{\lambda}_{i}^{*}+\alpha\left(\ddot{\mathbf{\Phi}}+2 \xi \omega \dot{\boldsymbol{\Phi}}+\omega^{2} \boldsymbol{\Phi}\right)
\end{aligned}
$$

where $\boldsymbol{\lambda}^{*}$ are the $m$ modified Lagrange multipliers and subscript $i$ stands for the iteration number. If the multipliers are updated only once in each integration time-step, then this formulation is equivalent to the penalty one in Eq. (5). Position-, velocity-, and acceleration-level massorthogonal projections were also introduced in [6] to ensure an accurate satisfaction of the kinematic constraints.

\subsection{Formulation based on Hamilton's canonical equations}

Formulations based on Hamilton's canonical equations constitute an alternative approach to the classical, acceleration-based augmented Lagrangian algorithms. They introduce the conjugate or canonical momenta $\mathbf{p}=\partial L / \partial \dot{\mathbf{q}}$, where $L$ is the system Lagrangian, as system variables besides the generalized coordinates $\mathbf{q}$ [16]. With the definition of the Hamiltonian $H=\mathbf{p}^{\mathrm{T}} \dot{\mathbf{q}}-L$ the canonical equations for a constrained system can be written as [2]

$$
\dot{\mathbf{q}}=\frac{\partial H}{\partial \mathbf{p}} ; \quad-\dot{\mathbf{p}}=\frac{\partial H}{\partial \mathbf{q}}-\mathbf{f}_{n c}+\boldsymbol{\Phi}_{\mathbf{q}}^{\mathrm{T}} \boldsymbol{\lambda}
$$

where $\mathbf{f}_{n c}$ are the non-conservative forces applied to the system. Following a procedure similar to the one described in [4], an augmented Lagrangian algorithm can be developed from Hamilton's equations. The system Lagrangian is defined to include the velocity-level kinematic constraints, as well as fictitious potential and kinetic energy terms, and a set of dissipative forces is added to the formulation [2]. The generalized velocities $\dot{\mathbf{q}}$ can then be obtained from the resulting expression

$$
\left(\mathbf{M}+\boldsymbol{\Phi}_{\mathbf{q}}^{\mathrm{T}} \alpha \boldsymbol{\Phi}_{\mathbf{q}}\right) \dot{\mathbf{q}}=\mathbf{p}-\boldsymbol{\Phi}_{\mathbf{q}}^{\mathrm{T}} \alpha\left(\boldsymbol{\Phi}_{t}+2 \xi \omega \boldsymbol{\Phi}+\omega^{2} \int_{t_{0}}^{t} \boldsymbol{\Phi} d t\right)-\boldsymbol{\Phi}_{\mathbf{q}}^{\mathrm{T}} \boldsymbol{\sigma}
$$


where $t_{0}$ is the starting time of the motion and $\sigma$ are the formulation multipliers, which verify $\dot{\boldsymbol{\sigma}}=\boldsymbol{\lambda}$. The time derivatives of the canonical momenta can be explicitly obtained from equation

$$
\dot{\mathbf{p}}=\mathbf{f}+\dot{\boldsymbol{\Phi}}_{\mathbf{q}}^{\mathrm{T}} \alpha\left(\dot{\boldsymbol{\Phi}}+2 \xi \omega \mathbf{\Phi}+\omega^{2} \int_{t_{0}}^{t} \mathbf{\Phi} d t\right)+\dot{\boldsymbol{\Phi}}_{\mathbf{q}}^{\mathrm{T}} \boldsymbol{\sigma}
$$

and the multipliers $\sigma$ are iteratively updated according to the expression

$$
\boldsymbol{\sigma}_{i+1}=\boldsymbol{\sigma}_{i}+\alpha\left(\dot{\boldsymbol{\Phi}}+2 \xi \omega \mathbf{\Phi}+\omega^{2} \int_{t_{0}}^{t} \boldsymbol{\Phi} d t\right)
$$

The algorithm in Eqs. (8)-(10) is also equivalent to a penalty method if the number of updates of the multipliers in each evaluation of $\dot{\mathbf{q}}$ is set to one.

The fact that the constraint reactions $\lambda$ are the derivatives with respect to time of the multipliers $\sigma$ has two important consequences. First, it is possible to select any arbitrary values of $\boldsymbol{\sigma}$ for the initial conditions of the simulation, at $t=0$. Once $\sigma$ is set, the initial momenta can be evaluated making use of Eq. (8). Second, the formulation multipliers $\sigma$ represent the integral over the time domain of the constraint reactions $\lambda$. This means that it is possible that some elements of $\boldsymbol{\sigma}$ grow indefinitely as the simulation progresses, e.g., when the corresponding elements of $\boldsymbol{\lambda}$ do not change sign. Such an issue can eventually cause the simulation to fail and must be avoided resetting the multipliers. When resetting takes place, the canonical momenta $\mathbf{p}$ and their derivatives $\dot{\mathbf{p}}$ must be re-evaluated to match the new values of $\sigma$.

\section{Implementation of the methods following a Newton-Raphson scheme}

The methods described in Section 2 can be used together with the numerical integrator formulas according to a fixed point integration scheme. Given the state of a mechanism at time-step $k, \mathbf{q}_{k}$ and $\dot{\mathbf{q}}_{k}$, Eqs. (5) or (6) can be used to evaluate the accelerations $\ddot{\mathbf{q}}_{k}$. These can be subsequently integrated to obtain the positions and velocities in the next time-step. For instance, with the well-known forward Euler explicit integration scheme, these would be evaluated as

$$
\mathbf{q}_{k+1}=\mathbf{q}_{k}+h \dot{\mathbf{q}}_{k} ; \quad \dot{\mathbf{q}}_{k+1}=\dot{\mathbf{q}}_{k}+h \ddot{\mathbf{q}}_{k} ;
$$


where $h$ is the integration step-size. A similar procedure can be adopted with the formulation based on Hamilton's equations, with $\mathbf{q}$ and $\mathbf{p}$ as variables. With Lagrangian methods, a semiimplicit integration formula can be used as well

$$
\mathbf{q}_{k+1}=\mathbf{q}_{k}+h \dot{\mathbf{q}}_{k+1} ; \quad \dot{\mathbf{q}}_{k+1}=\dot{\mathbf{q}}_{k}+h \ddot{\mathbf{q}}_{k} ;
$$

However, adopting a Newton-Raphson solution scheme instead of the fixed point one can be advantageous in terms of efficiency and stability [10]. This requires the introduction of the numerical integrator formulas in the equations of motion.

\subsection{Augmented Lagrangian methods}

The augmented Lagrangian algorithm in Eqs. (6) can be combined with the Newmark numerical integration formulas [28] to obtain a Newton-Raphson iterative scheme. The integration formulas are

$$
\begin{aligned}
& \dot{\mathbf{q}}_{k+1}=\frac{\gamma}{\beta h} \mathbf{q}_{k+1}-\widehat{\dot{\mathbf{q}}}_{k} ; \quad \text { where } \widehat{\hat{\mathbf{q}}}_{k}=\frac{\gamma}{\beta h} \mathbf{q}_{k}+\left(\frac{\gamma}{\beta}-1\right) \dot{\mathbf{q}}_{k}+h\left(\frac{\gamma}{2 \beta}-1\right) \ddot{\mathbf{q}}_{k} \\
& \ddot{\mathbf{q}}_{k+1}=\frac{1}{\beta h^{2}} \mathbf{q}_{k+1}-\widehat{\ddot{\mathbf{q}}}_{k} ; \quad \text { where } \widehat{\ddot{\mathbf{q}}}_{k}=\frac{1}{\beta h^{2}} \mathbf{q}_{k}+\frac{1}{\beta h} \dot{\mathbf{q}}_{k}+\left(\frac{1}{2 \beta}-1\right) \ddot{\mathbf{q}}_{k}
\end{aligned}
$$

where $\beta$ and $\gamma$ are scalar parameters, and subscript $k$ denotes the time-step. The well-known trapezoidal rule method is a particular case of the Newmark formulas in which $\beta=0.25$ and $\gamma=0.5$

Introducing the equations of the integrator (13) in the dynamics equations (6) and establishing the equilibrium at time-step $k+1$, a system of nonlinear equations is obtained

$$
\begin{aligned}
\mathbf{g}(\mathbf{q}, \dot{\mathbf{q}}) & =\left(\mathbf{M}+\boldsymbol{\Phi}_{\mathbf{q}}^{\mathrm{T}} \alpha \boldsymbol{\Phi}_{\mathbf{q}}\right)\left(\frac{1}{\beta h^{2}} \mathbf{q}_{k+1}-\widehat{\ddot{\mathbf{q}}}_{k}\right)-\mathbf{f} \\
& +\boldsymbol{\Phi}_{\mathbf{q}}^{\mathrm{T}} \alpha\left[\dot{\boldsymbol{\Phi}}_{\mathbf{q}}\left(\frac{\gamma}{\beta h} \mathbf{q}_{k+1}-\widehat{\dot{\mathbf{q}}}_{k}\right)+\dot{\boldsymbol{\Phi}}_{t}+2 \omega \xi\left(\boldsymbol{\Phi}_{\mathbf{q}}\left(\frac{\gamma}{\beta h} \mathbf{q}_{k+1}-\widehat{\dot{\mathbf{q}}}_{k}\right)+\boldsymbol{\Phi}_{t}\right)+\omega^{2} \boldsymbol{\Phi}\right] \\
& +\boldsymbol{\Phi}_{\mathbf{q}}^{\mathrm{T}} \boldsymbol{\lambda}_{k+1}^{*}=\mathbf{0}
\end{aligned}
$$


Such a system can be solved by means of a Newton-Raphson iteration scheme in the form

$$
\begin{aligned}
& {\left[\frac{d \mathbf{g}(\mathbf{q}, \dot{\mathbf{q}})}{d \mathbf{q}}\right]_{i} \Delta \mathbf{q}_{i+1}=-[\mathbf{g}(\mathbf{q}, \dot{\mathbf{q}})]_{i}} \\
& \mathbf{q}_{i+1}=\mathbf{q}_{i}+\Delta \mathbf{q}_{i+1}
\end{aligned}
$$

where $i$ stands for the iteration step. The tangent matrix of the system is obtained upon differentiation of Eq. (14) with respect to the generalized coordinates q. In this differentiation process, terms $\left(\mathbf{\Phi}_{\mathbf{q}}\right)_{\mathbf{q}}$ and $\left(\dot{\boldsymbol{\Phi}}_{\mathbf{q}}\right)_{\mathbf{q}}$ are neglected, as their contribution is usually small in comparison with the other terms. Moreover, the formulation multipliers $\lambda^{*}$ are considered to be independent from the problem variables. With these assumptions, after scaling, the expression of the tangent matrix is

$$
\begin{aligned}
{\left[\frac{d \mathbf{g}(\mathbf{q}, \dot{\mathbf{q}})}{d \mathbf{q}}\right] \cong } & \mathbf{M}+\beta h^{2} \mathbf{K}+\gamma h \mathbf{C}+\mathbf{\Phi}_{\mathbf{q}}^{\mathrm{T}} \alpha \mathbf{\Phi}_{\mathbf{q}}\left(1+2 \omega \xi \gamma h+\omega^{2} \beta h^{2}\right) \\
& +\boldsymbol{\Phi}_{\mathbf{q}}^{\mathrm{T}} \alpha \dot{\boldsymbol{\Phi}}_{\mathbf{q}} \gamma h+\boldsymbol{\Phi}_{\mathbf{q}}^{\mathrm{T}} \alpha\left(\beta h^{2}\left(\dot{\boldsymbol{\Phi}}_{t}\right)_{\mathbf{q}}+2 \omega \xi \beta h^{2}\left(\mathbf{\Phi}_{t}\right)_{\mathbf{q}}\right)
\end{aligned}
$$

where $\mathbf{C}=-\partial \mathbf{f} / \partial \dot{\mathbf{q}}$ and $\mathbf{K}=-\partial \mathbf{f} / \partial \mathbf{q}$. The residual takes the form

$$
\mathbf{g}(\mathbf{q}, \dot{\mathbf{q}})=\beta h^{2}\left(\mathbf{M} \ddot{\mathbf{q}}-\mathbf{f}+\boldsymbol{\Phi}_{\mathbf{q}}^{\mathrm{T}} \alpha\left(\ddot{\mathbf{\Phi}}+2 \omega \xi \dot{\boldsymbol{\Phi}}+\omega^{2} \mathbf{\Phi}\right)+\boldsymbol{\Phi}_{\mathbf{q}}^{\mathrm{T}} \boldsymbol{\lambda}^{*}\right)
$$

where all terms are evaluated at time-step $k+1$.

The leading matrix in Eq. (16), in general, is not symmetric. This may slow down the solution of Eq. (15), as it impedes the use of several efficient algorithms, such as those that involve Cholesky factorizations. In [6], mass-orthogonal projections were used to enforce the satisfaction of kinematic constraints at the configuration, velocity, and acceleration levels. It is then possible to assume that the constraints are exactly fulfilled at some of these levels, which enables one to remove the corresponding terms $\Phi$, $\dot{\Phi}$, or $\ddot{\boldsymbol{\Phi}}$ from the dynamics equations (6a). An index-1 Newton-Raphson implementation with position and velocity projections was described in [10]. To obtain this algorithm it was assumed that the projections enforced $\mathbf{\Phi}=\mathbf{0}$ and $\dot{\boldsymbol{\Phi}}=\mathbf{0}$ and only the term $\ddot{\Phi}$ had to be considered in the equations of motion. In the same paper, an index-3 approach with velocity and acceleration projections was also developed. Considering that $\dot{\Phi}=\mathbf{0}$ and $\ddot{\boldsymbol{\Phi}}=\mathbf{0}$ allows one to write Eq. (14) as

$$
\mathbf{g}(\mathbf{q}, \dot{\mathbf{q}})=\mathbf{M} \mathbf{q}_{k+1}+\beta h^{2} \mathbf{\Phi}_{\mathbf{q}}^{\mathrm{T}}\left(\boldsymbol{\lambda}_{k+1}^{*}+\alpha \boldsymbol{\Phi}_{k+1}\right)-\beta h^{2} \mathbf{f}_{k+1}-\beta h^{2} \mathbf{M} \widehat{\ddot{\mathbf{q}}}_{k}=\mathbf{0}
$$


The leading matrix in Eq. (15) can be approximated as [11]

$$
\left[\frac{d \mathbf{g}(\mathbf{q}, \dot{\mathbf{q}})}{d \mathbf{q}}\right] \cong \mathbf{M}+\gamma h \mathbf{C}+\beta h^{2}\left(\mathbf{\Phi}_{\mathbf{q}}^{\mathrm{T}} \alpha \mathbf{\Phi}_{\mathbf{q}}+\mathbf{K}\right)
$$

and the Lagrange multipliers can be updated during the iterative process in Eq. (15) as

$$
\boldsymbol{\lambda}_{i+1}^{*}=\boldsymbol{\lambda}_{i}^{*}+\alpha \boldsymbol{\Phi}_{i}
$$

The index-3 augmented Lagrangian formulation (ALi3) described by Eqs. (18)-(20) with velocity and acceleration projections features excellent robustness and efficiency properties and it has been successfully used in real-time simulation of medium-size and large multibody systems [13], [18].

\subsection{Formulation based on Hamilton's canonical equations}

The algorithm in Eqs. (8)-(10) can also take a Newton-Raphson iterative scheme in which the positions $\mathbf{q}$ and the canonical momenta $\mathbf{p}$ are treated as the primary variables of the numerical integration. The expression of the trapezoidal rule can be particularized in this case to consider only first order derivatives as

$$
\begin{aligned}
\dot{\mathbf{q}}_{k+1} & =\frac{2}{h} \mathbf{q}_{k+1}-\widehat{\dot{\mathbf{q}}}_{k} ; \quad \text { where } \widehat{\dot{\mathbf{q}}}_{k}=\frac{2}{h} \mathbf{q}_{k}+\dot{\mathbf{q}}_{k} \\
\dot{\mathbf{p}}_{k+1} & =\frac{2}{h} \mathbf{p}_{k+1}-\widehat{\dot{\mathbf{p}}}_{k} ; \quad \text { where } \widehat{\dot{\mathbf{p}}}_{k}=\frac{2}{h} \mathbf{p}_{k}+\dot{\mathbf{p}}_{k}
\end{aligned}
$$

Introducing the equations of the integrator (21) in the algorithm equations (8) and (9) and establishing the equilibrium at time-step $k+1$, a system of nonlinear equations is obtained

$$
\mathbf{g}_{h}(\mathbf{y})=\left[\begin{array}{l}
\mathbf{g}_{1}(\mathbf{y}) \\
\mathbf{g}_{2}(\mathbf{y})
\end{array}\right]=\mathbf{0} ; \quad \text { where } \mathbf{y}=\left[\begin{array}{l}
\mathbf{q} \\
\mathbf{p}
\end{array}\right]
$$

The expressions of functions $\mathbf{g}_{1}$ and $\mathbf{g}_{2}$ are

$$
\begin{aligned}
\mathbf{g}_{1}= & \mathbf{0}=\left(\mathbf{M}+\boldsymbol{\Phi}_{\mathbf{q}}^{\mathrm{T}} \alpha \boldsymbol{\Phi}_{\mathbf{q}}\right) \mathbf{q}_{k+1} \\
& -\frac{h}{2}\left[\left(\mathbf{M}+\boldsymbol{\Phi}_{\mathbf{q}}^{\mathrm{T}} \alpha \mathbf{\Phi}_{\mathbf{q}}\right) \widehat{\dot{\mathbf{q}}}_{k}+\mathbf{p}_{k+1}-\boldsymbol{\Phi}_{\mathbf{q}}^{\mathrm{T}} \alpha\left(\boldsymbol{\Phi}_{t}+2 \xi \omega \boldsymbol{\Phi}+\omega^{2} \int_{t_{0}}^{t} \boldsymbol{\Phi} d t\right)-\boldsymbol{\Phi}_{\mathbf{q}}^{\mathrm{T}} \boldsymbol{\sigma}\right]
\end{aligned}
$$


and

$$
\begin{aligned}
\mathbf{g}_{2}= & \mathbf{0}=\mathbf{p}_{k+1} \\
& -\frac{h}{2}\left[\hat{\mathbf{p}}_{k}+\mathbf{f}+\dot{\mathbf{\Phi}}_{\mathbf{q}}^{\mathrm{T}} \alpha\left(\boldsymbol{\Phi}_{\mathbf{q}}\left(\frac{2}{h} \mathbf{q}_{k+1}-\widehat{\mathbf{q}}_{k}\right)+\boldsymbol{\Phi}_{t}+2 \xi \omega \mathbf{\Phi}+\omega^{2} \int_{t_{0}}^{t} \boldsymbol{\Phi} d t\right)+\dot{\boldsymbol{\Phi}}_{\mathbf{q}}^{\mathrm{T}} \boldsymbol{\sigma}\right]
\end{aligned}
$$

The corresponding Newton-Raphson iteration scheme takes the form

$$
\begin{aligned}
& {\left[\frac{d \mathbf{g}_{h}(\mathbf{y})}{d \mathbf{y}}\right]_{i} \Delta \mathbf{y}_{i+1}=-\left[\mathbf{g}_{h}(\mathbf{y})\right]_{i}} \\
& \mathbf{y}_{i+1}=\mathbf{y}_{i}+\Delta \mathbf{y}_{i+1}
\end{aligned}
$$

As in the case of acceleration-based algorithms in Section 3.1, the iterative update of the formulation multipliers $\sigma$ in Eq. (10) can be carried out during the Newton-Raphson iteration, which increases the algorithm efficiency. The system tangent matrix is obtained upon differentiation of Eq. (22) with respect to the generalized coordinates $\mathbf{q}$ and the canonical momenta $\mathbf{p}$ as

$$
\left[\frac{d \mathbf{g}_{h}(\mathbf{y})}{d \mathbf{y}}\right]=\left[\begin{array}{cc}
\frac{d \mathbf{g}_{1}(\mathbf{y})}{d \mathbf{q}} & \frac{d \mathbf{g}_{1}(\mathbf{y})}{d \mathbf{p}} \\
\frac{d \mathbf{g}_{2}(\mathbf{y})}{d \mathbf{q}} & \frac{d \mathbf{g}_{2}(\mathbf{y})}{d \mathbf{p}}
\end{array}\right]
$$

With the same assumptions that were used to derive the tangent matrix in Eq. (16), the expression of the four terms in the tangent matrix is

$$
\begin{aligned}
& \frac{d \mathbf{g}_{1}(\mathbf{y})}{d \mathbf{q}} \cong \mathbf{M}+\mathbf{\Phi}_{\mathbf{q}}^{\mathrm{T}} \alpha\left((1+h \xi \omega) \mathbf{\Phi}_{\mathbf{q}}+\frac{h}{2}\left(\mathbf{\Phi}_{t}\right)_{\mathbf{q}}+\frac{h \omega^{2}}{2}\left(\int_{t_{0}}^{t} \mathbf{\Phi} d t\right)_{\mathbf{q}}\right) \\
& \frac{d \mathbf{g}_{1}(\mathbf{y})}{d \mathbf{p}} \cong-\frac{h}{2} \mathbf{I}_{n \times n} \\
& \frac{d \mathbf{g}_{2}(\mathbf{y})}{d \mathbf{q}} \cong-\frac{d \mathbf{f}}{d \mathbf{q}}-\dot{\boldsymbol{\Phi}}_{\mathbf{q}}^{\mathrm{T}} \alpha\left((1+h \xi \omega) \mathbf{\Phi}_{\mathbf{q}}+\frac{h}{2}\left(\mathbf{\Phi}_{t}\right)_{\mathbf{q}}+\frac{h \omega^{2}}{2}\left(\int_{t_{0}}^{t} \mathbf{\Phi} d t\right)_{\mathbf{q}}\right) \\
& \frac{d \mathbf{g}_{2}(\mathbf{y})}{d \mathbf{p}} \cong \mathbf{I}_{n \times n}
\end{aligned}
$$

where $\mathbf{I}_{n \times n}$ is the $n \times n$ identity matrix. The derivative of the forces vector with respect to the positions in Eq. (27c) can be developed as

$$
\frac{d \mathbf{f}}{d \mathbf{q}}=-\mathbf{K}-\frac{2}{h} \mathbf{C}
$$


and the integral term in Eqs. (27a) and (27c) can be evaluated as follows

$$
\left(\int_{t_{0}}^{t} \mathbf{\Phi} d t\right)_{\mathbf{q}}=\int_{t_{0}}^{t} \mathbf{\Phi}_{\mathbf{q}} d t \cong \sum_{j=0}^{k+1} h \mathbf{\Phi}_{\mathbf{q}_{j}}
$$

Finally, the residual terms $\mathbf{g}_{1}$ and $\mathbf{g}_{2}$ after scaling can be expressed more compactly in the form

$$
\begin{aligned}
& \mathbf{g}_{1}=\frac{h}{2}\left[\mathbf{M} \dot{\mathbf{q}}-\mathbf{p}+\boldsymbol{\Phi}_{\mathbf{q}}^{\mathrm{T}} \boldsymbol{\sigma}+\boldsymbol{\Phi}_{\mathbf{q}}^{\mathrm{T}} \alpha\left(\dot{\boldsymbol{\Phi}}+2 \xi \omega \boldsymbol{\Phi}+\omega^{2} \int_{t_{0}}^{t} \mathbf{\Phi} d t\right)\right] \\
& \mathbf{g}_{2}=\frac{h}{2}\left[\dot{\mathbf{p}}-\mathbf{f}-\dot{\boldsymbol{\Phi}}_{\mathbf{q}}^{\mathrm{T}} \boldsymbol{\sigma}-\dot{\boldsymbol{\Phi}}_{\mathbf{q}}^{\mathrm{T}} \alpha\left(\dot{\boldsymbol{\Phi}}+2 \xi \omega \boldsymbol{\Phi}+\omega^{2} \int_{t_{0}}^{t} \mathbf{\Phi} d t\right)\right]
\end{aligned}
$$

The solution of the linear system in Eq. (25) required by the Newton-Raphson iteration scheme can be carried out in an efficient way making use of the Schur complement. Instead of assembling the $2 n \times 2 n$ leading matrix in Eq. (25) and solving the resulting system of equations, the increment $\Delta \mathbf{q}_{i+1}$ can be obtained as

$$
\left(\frac{d \mathbf{g}_{1}}{d \mathbf{q}}-\frac{d \mathbf{g}_{1}}{d \mathbf{p}}\left(\frac{d \mathbf{g}_{2}}{d \mathbf{p}}\right)^{-1} \frac{d \mathbf{g}_{2}}{d \mathbf{q}}\right) \Delta \mathbf{q}_{i+1}=-\mathbf{g}_{1}+\frac{d \mathbf{g}_{1}}{d \mathbf{p}}\left(\frac{d \mathbf{g}_{2}}{d \mathbf{p}}\right)^{-1} \mathbf{g}_{2}
$$

Once $\Delta \mathbf{q}_{i+1}$ is known, the momenta increment $\Delta \mathbf{p}_{i+1}$ can be found as

$$
\Delta \mathbf{p}_{i+1}=\left(\frac{d \mathbf{g}_{2}}{d \mathbf{p}}\right)^{-1}\left(-\mathbf{g}_{2}-\frac{d \mathbf{g}_{2}}{d \mathbf{q}} \Delta \mathbf{q}_{i+1}\right)
$$

As shown by Eqs. (27b) and (27d), terms $d \mathbf{g}_{1} / d \mathbf{p}$ and $d \mathbf{g}_{2} / d \mathbf{p}$ are scaled $n \times n$ identity matrices, so Eqs. (31) and (32) become the simpler expressions

$$
\left(\frac{d \mathbf{g}_{1}}{d \mathbf{q}}+\frac{h}{2} \frac{d \mathbf{g}_{2}}{d \mathbf{q}}\right) \Delta \mathbf{q}_{i+1}=-\mathbf{g}_{1}-\frac{h}{2} \mathbf{g}_{2}
$$

and

$$
\Delta \mathbf{p}_{i+1}=-\mathbf{g}_{2}-\frac{d \mathbf{g}_{2}}{d \mathbf{q}} \Delta \mathbf{q}_{i+1}
$$

Thus, the solution to the $2 n \times 2 n$ system of equations in Eq. (25) can be obtained by solving the $n \times n$ system in Eq. (33) and subsequently updating the momenta increment $\Delta \mathbf{p}_{i+1}$ with Eq. (34). 


\section{Rank deficient Jacobian matrices and singular configurations}

The application of the Lagrangian approach to the dynamics equations (1), together with the differentiation of the kinematic constraints (1b) with respect to time, results in a system of linear equations that can be written as follows

$$
\left[\begin{array}{cc}
\mathbf{M} & \boldsymbol{\Phi}_{\mathbf{q}}^{\mathrm{T}} \\
\boldsymbol{\Phi}_{\mathbf{q}} & \mathbf{0}
\end{array}\right]\left[\begin{array}{c}
\ddot{\mathbf{q}} \\
\boldsymbol{\lambda}
\end{array}\right]=\left[\begin{array}{c}
\mathbf{f} \\
-\dot{\boldsymbol{\Phi}}_{\mathbf{q}} \dot{\mathbf{q}}-\dot{\boldsymbol{\Phi}}_{t}
\end{array}\right]
$$

If the Jacobian matrix $\mathbf{\Phi}_{\mathbf{q}}$ is rank deficient, the leading matrix of system (35) becomes singular. This means that an infinite set of values of the Lagrange multipliers $\lambda$ are valid solutions of the system and some additional assumptions must be made to choose one solution among all the possible ones [23]. Rank deficient Jacobian matrices can be the consequence of introducing redundant kinematic constraints. In this case, the Jacobian matrix is usually rank deficient during the whole motion of the system. Another possible cause is the existence of singular configurations in the workspace. When the system reaches one of these singularities, the number of degrees of freedom (DoF) suddenly increases and the Jacobian matrix undergoes a loss of rank.

All the algorithms presented in Section 2 are able to deal with rank deficient Jacobian matrices. The leading matrices in Eqs. (5), (6), and (8) are all symmetric and positive-definite, provided that an appropriate penalty factor $\alpha$ has been selected. Regarding the implementations in Newton-Raphson form in Section 3, this is only true in the case of the ALi3 formulation, Eq. (19). The leading matrices in Eqs. (16) and (26) are not symmetric in the general case. However, a rank deficient Jacobian matrix does not cause the algorithms to fail either. The use of the penalty technique in the algorithms discussed in this paper is equivalent to assuming a certain stiffness distribution within the system and this reduces the number of valid solutions for $\boldsymbol{\lambda}$ to only one [17]. However, it has been confirmed by experience that they may still suffer from numerical difficulties in the proximity of singular configurations.

\subsection{Benchmark examples}

Several multibody systems that feature redundant constraints and singular configurations can be found in the IFToMM library of benchmark problems [21]. Among these, we have selected three for the assessment of the dynamic formulations in Section 2. The first one is a six-link rectangu- 
lar Bricard mechanism (Fig. 1). This is a redundantly constrained, one-DoF mechanical system frequently used as benchmark problem, e.g., [19]. The set of kinematic constraints which are linearly dependent cannot be a priori identified, as it changes during motion. Therefore, redundant equations cannot be simply eliminated from the constraint set $\boldsymbol{\Phi}$, and the Jacobian matrix $\Phi_{\mathbf{q}}$ is permanently rank deficient. However, the system does not reach any singular configuration during its entire range of motion.

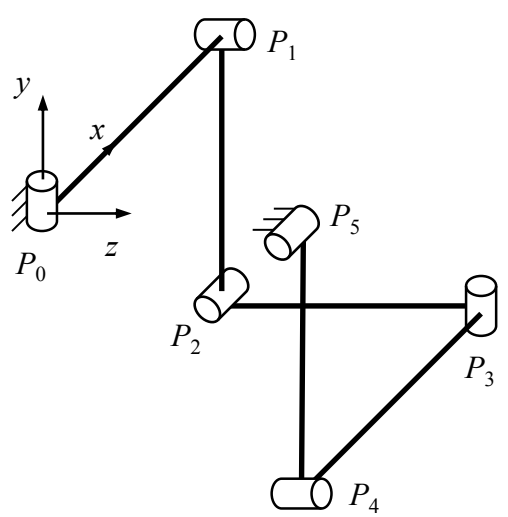

Figure 1: A six-link rectangular Bricard mechanism, a redundantly constrained multibody system without singular configurations.

Two planar linkages were chosen as examples of systems that undergo singular configurations: a slider-crank mechanism and a double four-bar linkage (Figs. 2 and 3). These were already used in [2] to discuss the performance of augmented Lagrangian formulations in the simulation of systems with singular configurations. Both are made up of rods of length $l=1$ $\mathrm{m}$ with a uniformly distributed mass $m_{b}=1 \mathrm{~kg}$ and a square cross section of width $r=0.1 \mathrm{~m}$, connected by revolute joints. Gravity $\left(g=9.81 \mathrm{~m} / \mathrm{s}^{2}\right)$ acts in the negative direction of the $y$-axis in the three examples.

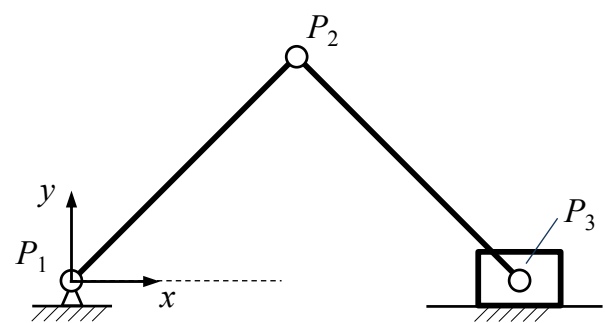

Figure 2: A slider-crank mechanism.

The forward-dynamics simulation of the motion of the Bricard mechanism can be used to show that the augmented Lagrangian formulations described in Sections 2 and 3 are able to successfully deal with rank deficient Jacobian matrices derived from the presence of redundant constraints. Conversely, numerical difficulties were observed in some cases during the simulation 


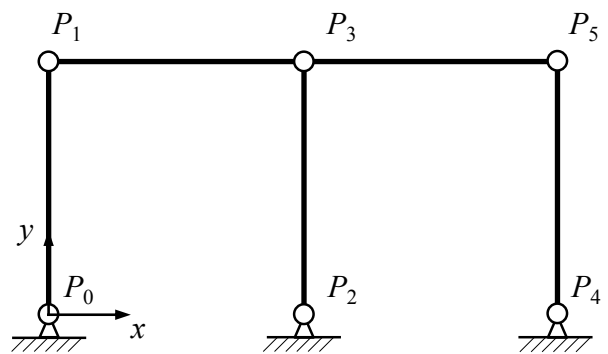

Figure 3: A double four-bar linkage.

of the slider-crank and the four-bar linkages when they were near a singular configuration.

\subsection{Behaviour of the formulations in the neighbourhood of a singular configura- tion}

The slider-crank mechanism in Fig. 2 is in a singular configuration when its two rods are aligned on the global $y$-axis. The linkage has one DoF during the rest of its motion, but at this configuration a new degree of freedom instantaneously appears. The singular configuration is in fact a bifurcation point, after which the system can continue its slider-crank motion or start to behave as a simple pendulum with point $P_{3}$ stopped at the global origin of coordinates. Both motions are actually possible when the linkage is exactly in the singular configuration and momentarily becomes a two-DoF system. The singular configuration for the four-bar linkage (Fig. 3) happens when all the links are aligned on the global $x$-axis; again, we have a bifurcation point at which three alternative motions are simultaneously feasible.

\subsubsection{Change in the subspace of admissible motion in singular configurations}

It can be useful to decompose the system velocities into its components contained in the subspaces of admissible and constrained motion [7], [8], [24] to highlight the role of singular configurations as bifurcation points. Given a mechanical system described with a set of $n$ generalized velocities $\dot{\mathbf{q}}$, the $m$ kinematic constraints at the velocity level represent a velocity transformation that can be used to define the subspace of constrained motion (SCM)

$$
\Phi_{\mathbf{q}} \dot{\mathbf{q}}=\mathbf{u}_{c}
$$


where $\mathbf{u}_{c}$ is a set of $m$ velocity components with respect to a local basis of the SCM. The dimension of the SCM is the rank of the Jacobian matrix $\mathbf{\Phi}_{\mathbf{q}}$, so this subspace will be $m$ dimensional if the kinematic constraints are linearly independent. The subspace of admissible motion (SAM) complements the SCM. The system velocities can be decomposed into two components as $\dot{\mathbf{q}}=\dot{\mathbf{q}}_{a}+\dot{\mathbf{q}}_{c}$ where $\dot{\mathbf{q}}_{a}$ is the set of generalized velocities admissible with the velocitylevel constraints in Eq. (3); $\dot{\mathbf{q}}_{c}$ is the velocity set which is not admissible with the constraints, i.e. constraint violations.

The slider-crank example can be modelled with a set of three planar natural coordinates $\mathbf{q}^{s c}$ composed of the $x$ - and $y$-coordinates of point $P_{2}, x_{2}$ and $y_{2}$, and the $x$-coordinate of point $P_{3}, x_{3}$. Two kinematic constraints, enforcing constant distances between the tips of the rods, are necessary to ensure the correct motion of the assembly. The corresponding equations at the velocity level are

$$
\dot{\mathbf{\Phi}}^{s c}=\left[\begin{array}{ccc}
2 x_{2} & 2 y_{2} & 0 \\
2\left(x_{2}-x_{3}\right) & 2 y_{2} & 2\left(x_{3}-x_{2}\right)
\end{array}\right]\left[\begin{array}{c}
\dot{x}_{2} \\
\dot{y}_{2} \\
\dot{x}_{3}
\end{array}\right]=\mathbf{\Phi}_{\mathbf{q}}^{s c} \dot{\mathbf{q}}^{s c}=\mathbf{0}
$$

where $\boldsymbol{\Phi}_{\mathbf{q}}^{s c}$ is the Jacobian matrix that corresponds to the two constant-distance constraints. Let us consider that at $t=0$ link $P_{1}-P_{2}$ is at an angle $\phi=\phi_{0}=\pi / 4$ with respect to the $x$-axis, and that $\dot{x}_{3}=-4 \mathrm{~m} / \mathrm{s}$. At time $t=t_{s}$ the system reaches a singular configuration, in which $\phi=\pi / 2$, $x_{2}=x_{3}=0$, and $y_{2}=l \mathrm{~m}$. For $t<t_{s}$, the Jacobian matrix $\boldsymbol{\Phi}_{\mathbf{q}}^{s c}$ has rank two and any admissible velocity set can be expressed as

$$
\dot{\mathbf{q}}_{a}^{s c}=\eta\left[\begin{array}{c}
1 \\
-x_{2} / y_{2} \\
x_{3} /\left(x_{3}-x_{2}\right)
\end{array}\right]
$$

where $\eta$ is a scalar. At $t=t_{s}$, the system is in a singular configuration, and the Jacobian matrix becomes

$$
\left.\boldsymbol{\Phi}_{\mathbf{q}}^{s c}\right|_{t_{s}}=\left[\begin{array}{lll}
0 & 2 l & 0 \\
0 & 2 l & 0
\end{array}\right]
$$

which is a rank-1 matrix. The SCM for this instant is a one-dimensional subspace. Consequently, 
the SAM has dimension two. Among the several alternatives to parametrize this subspace a possible one is

$$
\left.\dot{\mathbf{q}}_{a}^{s c}\right|_{t_{s}}=\eta_{1}\left[\begin{array}{c}
1 \\
0 \\
2
\end{array}\right]+\eta_{2}\left[\begin{array}{c}
1 \\
0 \\
0
\end{array}\right]=\eta_{1} \dot{\mathbf{q}}_{a 1}^{s c}+\eta_{2} \dot{\mathbf{q}}_{a 2}^{s c}
$$

where $\eta_{1}$ and $\eta_{2}$ are scalar parameters. Vector $\dot{\mathbf{q}}_{a 1}^{s c}$ corresponds to the slider-crank motion of the mechanism, while $\dot{\mathbf{q}}_{a 2}^{s c}$ represents a single pendulum motion with point $P_{3}$ fixed at the origin. The condition $x_{2}=x_{3}$ makes both branches simultaneously possible, so the velocity vector $\dot{\mathbf{q}}$ of the system can have components along both $\dot{\mathbf{q}}_{a 1}^{s c}$ and $\dot{\mathbf{q}}_{a 2}^{s c}$. However, when the system leaves the singular configuration at $t>t_{s}$ it reverts to a one-dimensional SAM, which will be either the slider-crank one compatible with $\dot{\mathbf{q}}_{a 1}^{s c}$ or the simple pendulum motion defined by $\dot{\mathbf{q}}_{a 2}^{s c}$, depending on how the numerical integration process proceeded at $t=t_{s}$.

This reasoning can be generalized to any one-DoF mechanical system. The introduction of an extra DoF at a singularity momentarily expands the set of admissible velocities, which becomes a linear combination of a velocity vector in continuity with the pre-existing system motion, $\dot{\mathbf{q}}_{a 1}$, and a new one $\dot{\mathbf{q}}_{a 2}$ which is also compatible with the constraints. Both components are only simultaneously admissible at the singular configuration; at this point $\dot{\mathbf{q}}_{a}=\eta_{1} \dot{\mathbf{q}}_{a 1}+\eta_{2} \dot{\mathbf{q}}_{a 2}$. No matter which component defines the motion after the singularity, the other one will become a violation of the velocity-level constraints (3). Augmented Lagrangian formulations based on penalty approaches transform the constraint violations into constraint reactions, as shown in Eq. (2). Accordingly, penalty-based formulations remove the velocity component along the no longer admissible direction by introducing an impact when the system leaves the singular configuration.

The forward-dynamics simulation of the slider-crank motion starting from the singular configuration supports the previous statements. As correctly pointed out in the literature, e.g., [2], the simulation can be started from a singularity because the formulations in Section 2 are able to find a solution for the dynamics equations even with a rank deficient Jacobian matrix. Here, the penalty formulation in Eq. (5) was used with a penalty factor $\alpha=10^{7}$, parameters $\omega=10$ and $\xi=1$, and the trapezoidal rule as integrator with a step-size $h=10^{-3}$ s. First, the initial velocity was made proportional to $\dot{\mathbf{q}}_{a 1}^{s c}$ by choosing $\eta_{1}=-2 \mathrm{~m} / \mathrm{s}$ and $\eta_{2}=0$. Afterwards, $\eta_{2}$ was given different non-zero values and the simulation was repeated for each of them. Fig. 4 shows that introducing a component of $\dot{\mathbf{q}}$ along $\dot{\mathbf{q}}_{a 2}^{s c}$ gives rise to impact forces in the constraint 


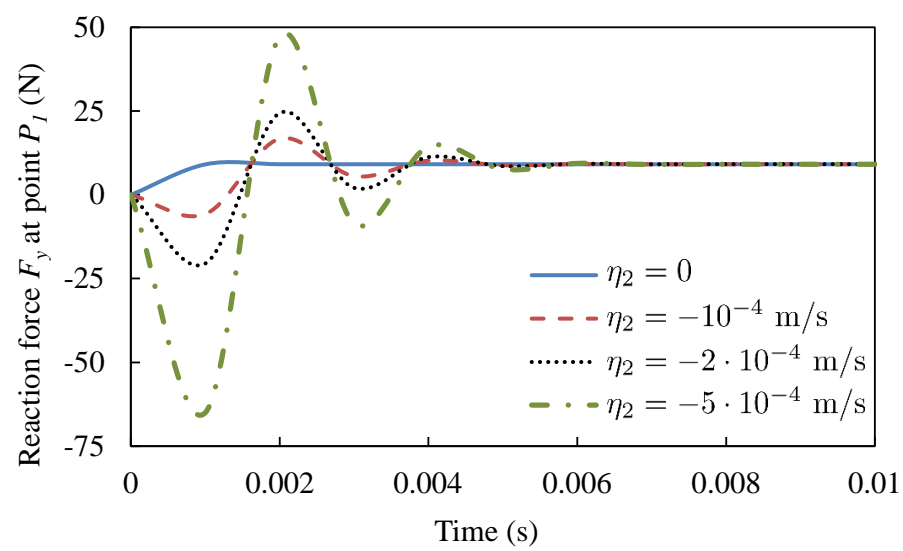

Figure 4: $y$ component of the reaction force at point $P_{1}$ during motion of the slider-crank mechanism, starting from a singular configuration, for different initial velocities.

reactions. Numerical experiments with the other formulations described in Section 2 showed the same behaviour.

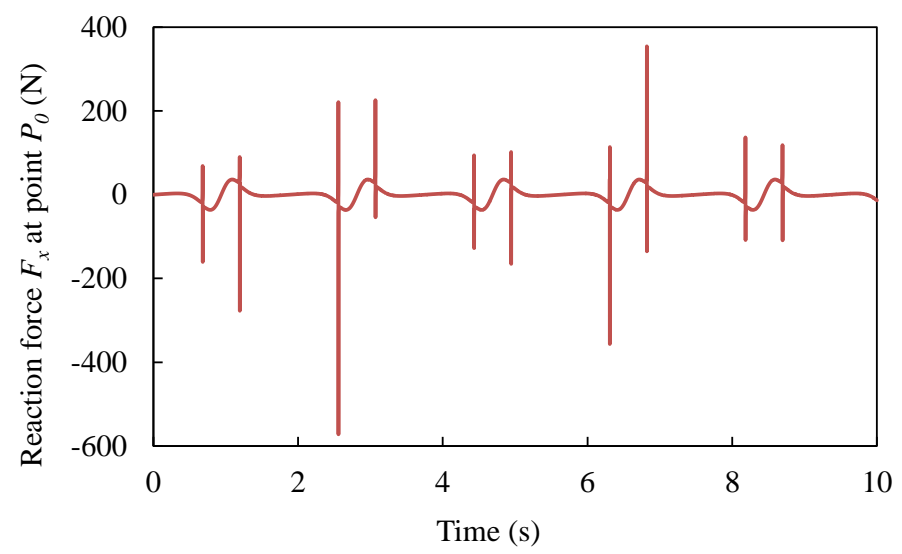

Figure 5: $x$ component of the reaction force at point $P_{0}$ during motion of the four-bar linkage, showing impacts when the system is near a singularity.

Moreover, the simulation of a $10 \mathrm{~s}$ motion of the four-bar linkage (Fig. 3) confirmed that the reaction force in the $x$-direction at point $P_{0}$ featured the same impact forces, as shown in Fig. 5. To obtain these results, the ALi3 formulation with velocity and acceleration projections, Eqs. (18)-(20), was used, with stringent convergence requirements to ensure that the constraint violations at the configuration and velocity levels remained close to machine precision. Similar force spikes can be observed in other publications in the literature, e.g., [9]. It should be stressed that the velocity component along $\dot{\mathbf{q}}_{a 2}$ cannot be eliminated by the velocity projections at the singular configuration, because it is not a violation of the constraints at that point. As expected, these impacts are not present in the simulation of redundantly constrained mechanisms without singular configurations, as in the case of the Bricard mechanism in Fig. 1. 


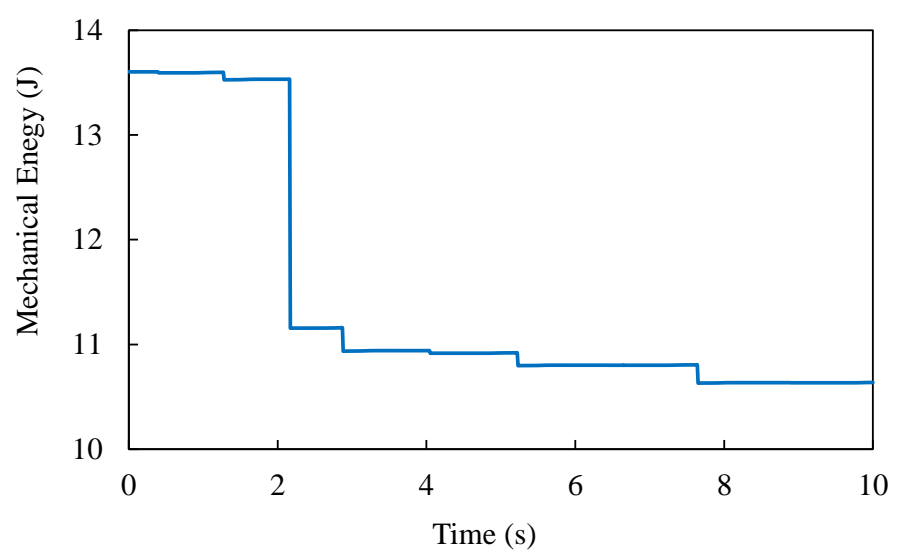

Figure 6: Mechanical energy of the slider-crank mechanism integrated with the explicit forward Euler method $(h=$ $\left.10^{-5} \mathrm{~s}\right)$ and the penalty formulation $\left(\alpha=10^{8}, \omega=10, \xi=1\right)$. A change of branch occurs at $t=2.17 \mathrm{~s}$.

The impact forces above described introduce a series of undesirable effects in the simulations if the numerical integrator and the formulation parameters are not properly selected. They generate discontinuities in the mechanical energy of the system, as shown in Fig. 6. Sometimes they can cause the mechanical system to undergo a change of branch when it leaves the singular configuration. In this case, a discontinuity in the motion takes place and the system velocities after the singularity are no longer in continuity with the pre-singularity motion compatible with $\dot{\mathbf{q}}_{a 1}$, but with the secondary one defined by $\dot{\mathbf{q}}_{a 2}$. In extreme cases they may bring about the failure of the simulation.

\subsubsection{Effect of configuration-level constraint violations}

The formulations in Sections 2 and 3 are rather robust and able to handle large impact forces during the pass through singularity. Numerical simulations showed that $\eta_{1}$ and $\eta_{2}$ need be of the same order of magnitude for a change of branch to take place in most cases. The exception is the index-3 augmented Lagrangian formulation. For example, starting the simulation of the slider-crank at the singular configuration with $\eta_{1}=-1 \mathrm{~m} / \mathrm{s}$ and $\eta_{2}=-5 \cdot 10^{-5} \mathrm{~m} / \mathrm{s}$ results in a pendulum motion after the singularity, with $\alpha=10^{9}$ and a step-size $h=10^{-3} \mathrm{~s}$. Such values of $\eta_{2}$ are usually not reached in practice because the velocity projections keep this component small during motion.

A violation of the configuration-level constraints, however, alters the expression of the Jacobian matrix $\boldsymbol{\Phi}_{\mathbf{q}}$ and changes the definition of the constrained and admissible subspaces of motion. A modification of the generalized coordinates not compatible with the constraints, $\epsilon$, 


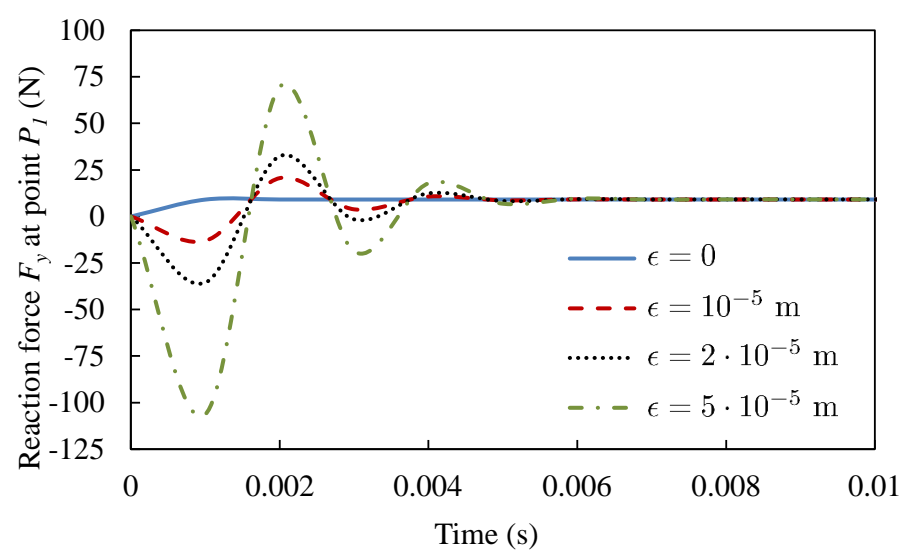

Figure 7: $y$ component of the reaction force at point $P_{1}$ during motion of the slider-crank mechanism, starting from a singular configuration, for different initial configuration-level constraint violations $\epsilon$.

makes the Jacobian matrix become $\tilde{\boldsymbol{\Phi}}_{\mathbf{q}}=\boldsymbol{\Phi}_{\mathbf{q}}(\mathbf{q}+\boldsymbol{\epsilon})$. In general, $\tilde{\boldsymbol{\Phi}}_{\mathbf{q}} \dot{\mathbf{q}} \neq \mathbf{0}$, even though the system velocities have theoretically correct values. This means that part of the admissible generalized velocities will be treated as velocity-level constraint violations, giving rise to the impact forces described in Section 4.2.1. Fig. 7 shows these impact forces in the simulation of the slider-crank mechanism with the penalty formulation and the same parameters of Section 4.2.1, starting from the singularity. A configuration error was introduced in the initial position by making $x_{2}=-\epsilon$ and $x_{3}=\epsilon$. Simulation showed that the effect of configuration-level constraint violations is much more critical than its velocity-level counterpart. For instance, an initial error in the order of $\epsilon=10^{-2} \mathrm{~m}$ is enough to trigger a branch change with $\eta_{2}=0$.

\section{Numerical results}
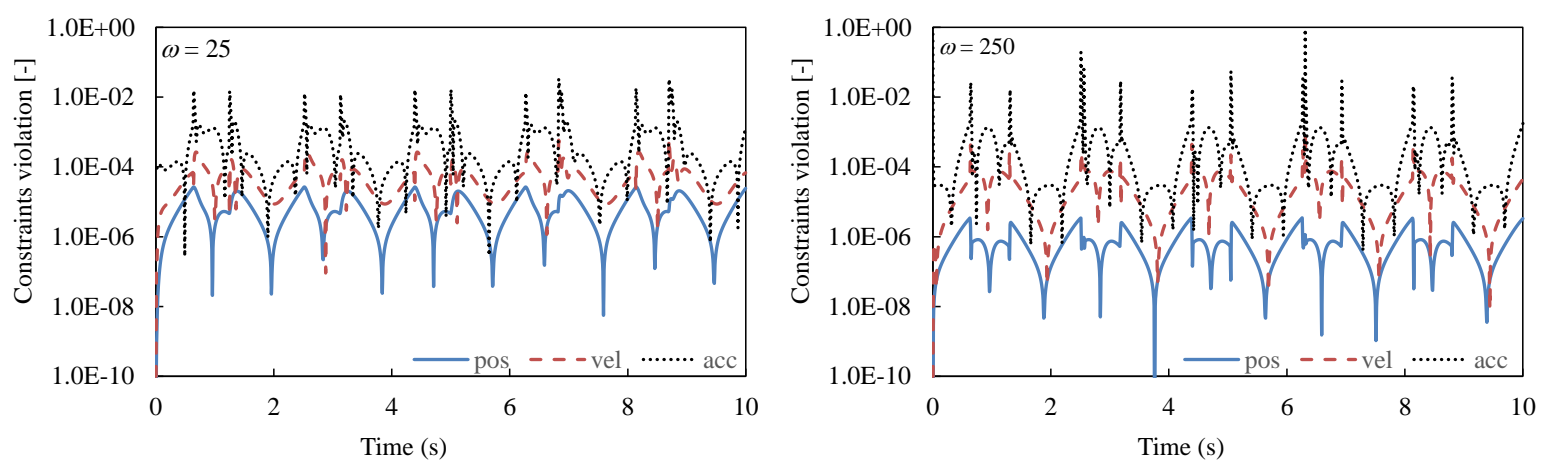

Figure 8: Constraint violations of the four-bar linkage during simulation with the augmented Lagrangian method and the trapezoidal rule in fixed point scheme $\left(\alpha=10^{7}, \xi=1, h=1 \mathrm{~ms}\right)$. Parameter $\omega$ was set to $\omega=25$ (left plot) and $\omega=250$ (right plot). 
The existence of singular configurations is not the result of a deficient modelling or the wrong choice of simulation strategy, but a property of some mechanisms. Even though a simulation algorithm be able to deal with rank deficient Jacobian matrices, the enlargement of the SAM in the singularity points described in Section 4.2.1 remains. In fact, all the methods mentioned in Sections 2 and 3 have been found to experience numerical problems and fail near singular configurations in the simulation of the slider-crank mechanism and the double four-bar linkage for certain combinations of their parameters.

The natural motion of a mechanism would keep the continuity of the velocities during the pass through the singularities. In other words, the ideal simulation of the system motion should not introduce impact forces in the reactions at the singular configurations. Conversely, large values of these impact forces may result in discontinuities in the mechanical energy, which can lead to changes of branch or the simulation failure if the algorithm is unable to recover from the impact. Keeping the violation of kinematic constraints low, especially the configuration-level ones, is a way to reduce the magnitude of the impact forces. This is in accordance with guidelines provided in the literature, e.g., [2], [5]. A simulation algorithm based on a penalty approach must therefore meet two requirements: good constraint stabilization, especially at the configuration level, and robustness to withstand impact forces. A correct adjustment of the penalty factor $\alpha$ and the stabilization parameters $\xi$ and $\omega$ is necessary to satisfy these requirements. In both the penalty and the augmented Lagrangian formulations, the constraint reactions are proportional to $\ddot{\Phi}, \dot{\Phi}$, and $\boldsymbol{\Phi}$ as shown in Eq. (2). Increasing $\omega$ assigns more weight to the configuration-level constraint violations, which helps to overcome singular configurations.

Figure 8 exemplifies the effect of parameter $\omega$ on the constraint violations during the motion of the four-bar linkage, simulated with the augmented Lagrangian method and the trapezoidal rule. The left plot on this figure was obtained with $\omega=25$. Increasing this parameter up to $\omega=$ 250 results in a better satisfaction of the configuration-level constraints, but it can be detrimental for the fulfilment of the velocity- and especially acceleration-level ones. The modification of $\omega$ did not significantly alter the elapsed time in the computations. To achieve a similar effect with the Hamiltonian formulations in Sections 2.3 and 3.2, term $2 \xi \omega \Phi$ must be predominant in Eq. (10), which can be achieved raising the value of $\xi$. 


\subsection{Performance comparison}

Forward-dynamics simulations of the motion of the four-bar linkage and the slider-crank mechanisms were carried out to compare the different formulations in terms of efficiency and robustness. The numerical experiments were performed in an Intel Core i7-4790K at $4.00 \mathrm{GHz}$. The methods were implemented in $\mathrm{C}++$ making use of the library routines described in [20].

Table 1: Best performances obtained with each formulation in a $10 \mathrm{~s}$ simulation of the slider-crank mechanism motion, for a maximum energy drift of $0.1 \mathrm{~J}$. The forward Euler integration formula was used in all cases.

\begin{tabular}{ccccccccc}
\hline & & & & & & \multicolumn{3}{c}{ elapsed time } \\
Formulation & Eqs. & Integrator & $h(\mathrm{~ms})$ & $\alpha$ & $\omega$ & $\xi$ & total (s) & per time-step $(\mu \mathrm{s})$ \\
\hline Penalty & $(5)$ & Explicit & 0.02 & $10^{8}$ & 25 & 1 & 1.53 & 3.1 \\
Aug. Lagrangian & $(6)$ & Explicit & 0.01 & $10^{7}$ & 10 & 1 & 3.83 & 3.8 \\
Penalty & $(5)$ & Semi-Implicit & 2 & $10^{8}$ & 250 & 1 & 0.02 & 4.0 \\
Aug. Lagrangian & $(6)$ & Semi-Implicit & 2 & $10^{8}$ & 250 & 1 & 0.03 & 6.0 \\
Aug. Hamiltonian & $(8)-(10)$ & - & 2 & $10^{9}$ & 0.1 & 1000 & 0.02 & 4.0 \\
\hline
\end{tabular}

Table 1 compares the performance of the formulations mentioned in Section 2 in a $10 \mathrm{~s}$ simulation of the slider-crank mechanism motion. Initially, $\operatorname{rod} P_{1}-P_{2}$ is at an angle $\varphi=45^{\circ}$ with respect to the $x$-axis and the velocity of point $P_{3}$ is $\dot{x}_{3}=-4 \mathrm{~m} / \mathrm{s}$. The single-step explicit and semi-implicit forward Euler formulas were used as integrators. For the penalty and the index-1 augmented Lagrangian formulation, $\omega$ and $\xi$ were automatically set to $\xi=1 / \sqrt{2}$ and $\omega=\sqrt{2} / h$ in a first approach [15]. These parameters were subsequently tuned to improve the simulation efficiency. A variation range was defined for parameters $\alpha, \omega$, and $\xi$. A grid of sampling points was built dividing these ranges in fixed intervals. A simulation of the test problem motion was run for each sampling point, keeping the integration step-size as large as possible while satisfying the requirement of keeping the mechanical energy drift below $0.1 \mathrm{~J}$. Fine tuning was performed for those combinations of parameters that performed best. This proved to be a time-consuming process with the penalty formulation, as energy conservation was noticeably affected by changes in the formulation parameters. On the other hand, the augmented Lagrangian method showed a much more consistent behaviour for a wider range of the parameters. Parameters $\omega$ and $\xi$ of the Hamiltonian formulation were initially set to penalize the configuration-level constraint violations at least 200 times more than the other terms in Eq. (10), and adjusted following a method similar to the one used in the Lagrangian case.

Results showed that the numerical integrator plays a key role in the efficiency of the simulations. The penalty and index-1 augmented Lagrangian methods required integration step-sizes 
as low as $10^{-5} \mathrm{~s}$ to meet the energy requirement with the explicit integration formula; the use of the semi-implicit integrator enabled the use of $2 \cdot 10^{-3} \mathrm{~s}$ step-sizes. The Hamiltonian algorithm was also able to complete the integration with $h=2 \cdot 10^{-3} \mathrm{~s}$. It is worth noting that the Hamiltonian equations are first-order, so the explicit and semi-explicit integration schemes reduced to the same formula in this case.

Table 2: Best performances obtained with each formulation in a $10 \mathrm{~s}$ simulation of the slider-crank mechanism motion, for a maximum energy drift of $0.001 \mathrm{~J}$. The selected integrators were the forward Euler (FE) and the semiimplicit forward Euler (FE, SI) methods, the trapezoidal rule (TR), and the trapezoidal rule with Newton-Raphson implementation (TR, NR).

\begin{tabular}{cccccccccc}
\hline & & & & & & \multicolumn{1}{c}{} & \multicolumn{2}{c}{ elapsed time } \\
Formulation & Integrator & Eqs. & Tolerance & $h$ (ms) & $\alpha$ & $\omega$ & $\xi$ & total (s) & per time-step $(\mu \mathrm{s})$ \\
\hline Penalty & FE, SI & $(5)$ & - & 0.02 & $10^{8}$ & 250 & 1 & 1.57 & 3.1 \\
Aug. Lagrangian & FE, SI & $(6)$ & - & 0.02 & $10^{7}$ & 250 & 1 & 1.95 & 3.9 \\
Aug. Hamiltonian & FE & $(8)-(10)$ & - & 0.02 & $10^{9}$ & 0.1 & 1000 & 2.10 & 4.2 \\
Penalty & TR & $(5)$ & $10^{-7}$ & 0.1 & $10^{7}$ & 1000 & 1 & 0.33 & 3.3 \\
Aug. Lagrangian & TR & $(6)$ & $10^{-7}$ & 1 & $10^{7}$ & 200 & 1 & 0.06 & 6.0 \\
Aug. Hamiltonian & TR & $(8)-(10)$ & $10^{-7}$ & 2 & $10^{9}$ & 0.1 & 2000 & 0.13 & 26.0 \\
Aug. Lagrangian & TR, NR & $(15)-(17)$ & $10^{-7}$ & 1 & $10^{7}$ & 500 & 0.7 & 0.07 & 7.0 \\
Aug. Hamiltonian & TR, NR & $(25)-(34)$ & $10^{-7}$ & 2 & $10^{8}$ & 1 & $10^{5}$ & 0.14 & 28.0 \\
ALi3 & TR, NR & $(18)-(20)$ & $10^{-5}$ & 1 & $10^{9}$ & - & - & 0.07 & 7.0 \\
\hline
\end{tabular}

Next, the simulations were repeated for a maximum admissible energy drift of $0.001 \mathrm{~J}$, as required by the problem definition in [21]. It was impossible to meet this requirement with the acceleration-based Lagrangian algorithms using the explicit forward Euler integrator with reasonable step-sizes. The semi-implicit forward Euler and the trapezoidal rule were used as alternatives. This latter integrator introduced an iterative process in each time-step. It was observed that this process may diverge in the proximity of a singularity. This required the detection of divergence and the interruption of the iteration for the simulation to proceed successfully in some cases. Results are summarized in Table 2. Similar results were obtained for a $10 \mathrm{~s} \mathrm{sim-}$ ulation of the motion of the double four-bar linkage and are shown in Table 3. In the initial configuration of this mechanism, rods $P_{0}-P_{1}, P_{2}-P_{3}$, and $P_{4}-P_{5}$ were parallel to the $y$-axis, and the velocity of points $P_{1}, P_{3}$, and $P_{5}$ was $\dot{x}_{1}=\dot{x}_{3}=\dot{x}_{5}=1 \mathrm{~m} / \mathrm{s}$. Both tables include the integrator tolerance when implicit integrators are used, i.e., the stopping criterion for the iteration process. This tolerance was defined as the maximum admissible norm of the difference between the generalized coordinates obtained in two consecutive iterations of the solver.

Numerical experiments confirmed that a robust and efficient performance in the simulation of systems with singular configurations depends not only on the selected dynamic formulation and the tuning of its parameters, but also on the numerical integration expressions. Forward 
Table 3: Best performances obtained with each formulation in the $10 \mathrm{~s}$ simulation of the double four-bar linkage motion, for a maximum energy drift of $0.1 \mathrm{~J}$. The selected integrators were the forward Euler (FE) and the semiimplicit forward Euler (FE, SI) methods, the trapezoidal rule (TR), and the trapezoidal rule with Newton-Raphson implementation (TR, NR).

\begin{tabular}{cccccccccc}
\hline & & & & & & & \multicolumn{3}{c}{ elapsed time } \\
Formulation & Integrator & Eqs. & Tolerance & $h$ (ms) & $\alpha$ & $\omega$ & $\xi$ & total (s) & per time-step $(\mu$ s) \\
\hline Penalty & FE & $(5)$ & - & 0.02 & $10^{7}$ & 30 & 1 & 2.50 & 5 \\
Aug. Lagrangian & FE & $(6)$ & - & 0.005 & $10^{7}$ & 10 & 1 & 12.21 & 6 \\
Penalty & FE, SI & $(5)$ & - & 1 & $10^{8}$ & 400 & 1 & 0.06 & 6 \\
Aug. Lagrangian & FE, SI & $(6)$ & - & 1 & $10^{8}$ & 400 & 1 & 0.08 & 8 \\
Aug. Hamiltonian & FE & $(8)-(10)$ & - & 1 & $10^{9}$ & 0.1 & 1000 & 0.07 & 7 \\
Penalty & TR & $(5)$ & $10^{-7}$ & 5 & $10^{8}$ & 25 & 1 & 0.04 & 20 \\
Aug. Lagrangian & TR & $(6)$ & $10^{-7}$ & 5 & $10^{8}$ & 20 & 1 & 0.05 & 25 \\
Aug. Hamiltonian & TR & $(8)-(10)$ & $10^{-7}$ & 5 & $10^{9}$ & 0.1 & 1000 & 0.10 & 50 \\
Aug. Lagrangian & TR, NR & $(15)-(17)$ & $10^{-7}$ & 5 & $10^{7}$ & 100 & 1 & 0.05 & 25 \\
Aug. Hamiltonian & TR, NR & $(25)-(34)$ & $10^{-7}$ & 5 & $10^{8}$ & 1 & $10^{4}$ & 0.08 & 40 \\
ALi3 & TR, NR & $(18)-(20)$ & $10^{-7}$ & 10 & $10^{9}$ & - & - & 0.02 & 20 \\
\hline
\end{tabular}

Euler formulas performed acceptably well when the admissible energy drift was relatively high, $\Delta E=0.1 \mathrm{~J}$; when penalty and Lagrangian formulations were used, choosing a semi-implicit integration scheme over an explicit one considerably improved simulation efficiency. Implicit, iterative integrators showed a clearly superior performance when the maximum admissible drift was brought down to $0.001 \mathrm{~J}$.

\subsection{Fixed point and Newton-Raphson implementations}

Tables 2 and 3 also show that the efficiency of the Newton-Raphson implementations is comparable to that of their fixed point counterparts in the studied examples. However, the former showed a much more robust response to variations in the formulation parameters during the same tests. This is a significant advantage, because tuning up their values to obtain optimum performance can be a time-consuming and cumbersome task.

As an example, Fig. 9 shows the energy drift obtained during the simulation of the four-bar linkage motion with the penalty formulation and the trapezoidal rule in fixed point scheme. The penalty factor $\alpha$ was varied in a range from $10^{6}$ to $10^{8.5}$. Parameter $\omega$ was selected as variable too because it determines the relative weight of the configuration-level constraint violations $\mathbf{\Phi}$ in the penalty system of Eq. (2). Parameter $\xi$ was set to 1 . White dots in the plot indicate that the energy error was larger than $0.125 \mathrm{~J}$, and so the simulation failed to meet the maximum energy drift requirement. For an integration step-size $h=5 \mathrm{~ms}$, only values from a small region 


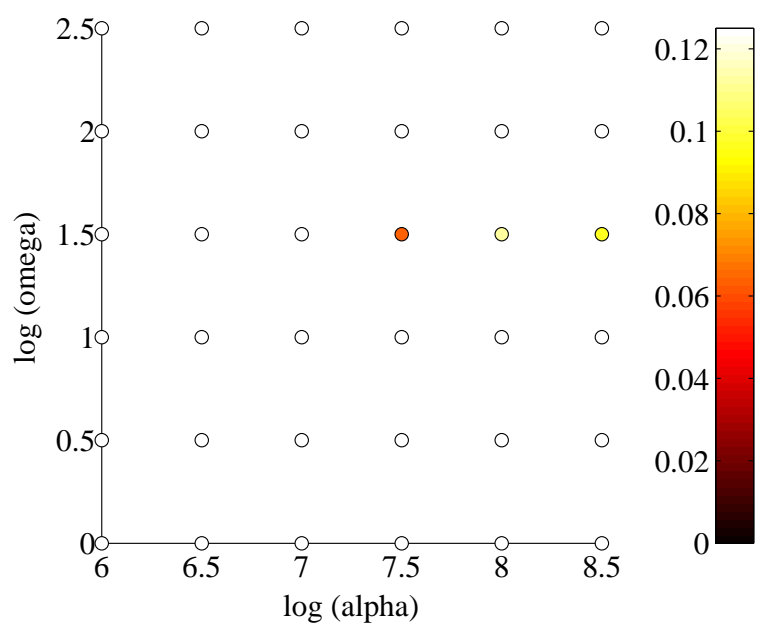

Figure 9: Energy drift during the simulation of the double four-bar linkage, with the penalty formulation, for different values of the penalty factor $\alpha$ and parameter $\omega$. The trapezoidal rule in fixed point scheme was used as integrator, with $h=5 \mathrm{~ms}$.

in the $\alpha-\omega$ plane resulted in a valid simulation.

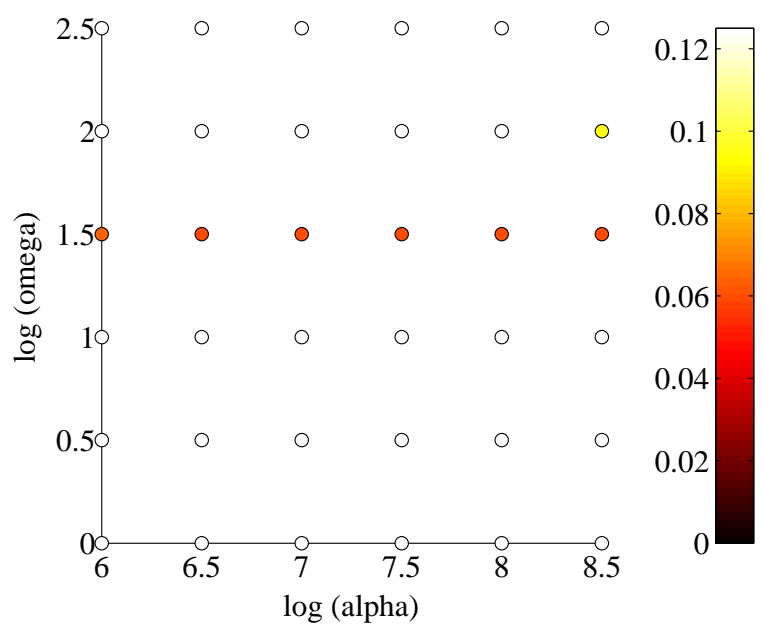

Figure 10: Energy drift during the simulation of the double four-bar linkage, with the augmented Lagrangian formulation, for different values of the penalty factor $\alpha$ and parameter $\omega$. The trapezoidal rule in fixed point scheme was used as integrator, with $h=5 \mathrm{~ms}$.

Replacing the penalty formulation with the augmented Lagrangian algorithm in Eq. (6) enlarged the range of penalty factors $\alpha$ with which correct simulations were obtained, as shown in Fig. 10. Still, $\omega$ had to be carefully adjusted.

The use of the augmented Lagrangian method in Newton-Raphson form made it possible to meet the energy drift requirement for a wider range of the $\alpha$ and $\omega$ parameters, as shown in Fig. 11. With this approach, the simulation behaviour was practically independent from the penalty factor within the studied range. Similar plots were obtained in the simulation of the slider-crank example. 


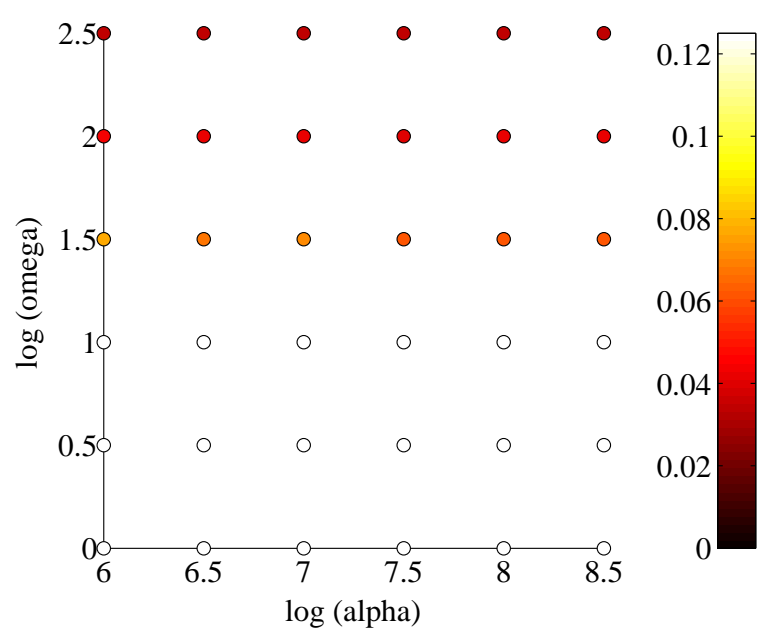

Figure 11: Energy drift during the simulation of the double four-bar linkage, with the augmented Lagrangian formulation, for different values of the penalty factor $\alpha$ and parameter $\omega$. The trapezoidal rule in Newton-Raphson scheme was used as integrator, with $h=5 \mathrm{~ms}$.

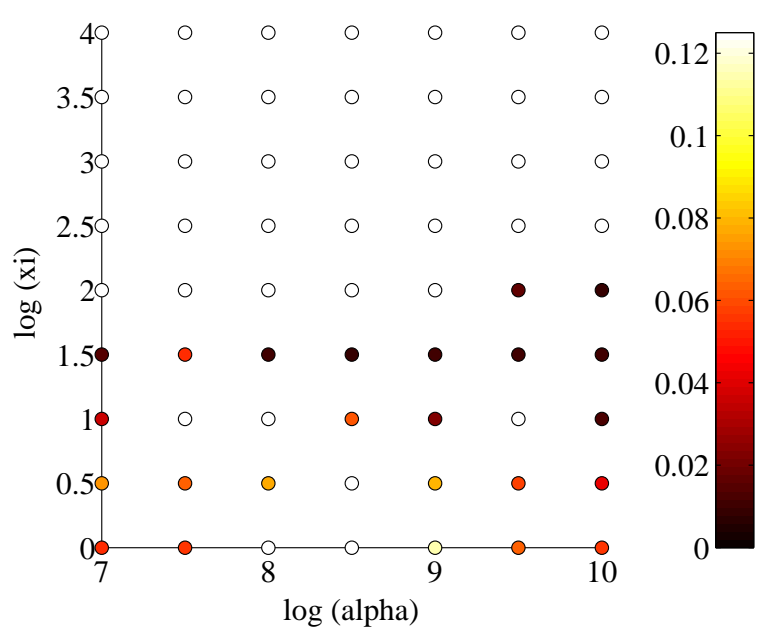

Figure 12: Energy drift during the simulation of the double four-bar linkage, with the augmented Hamiltonian formulation, for different values of the penalty factor $\alpha$ and parameter $\xi$. The trapezoidal rule in fixed point scheme was used as integrator, with $h=5 \mathrm{~ms}$.

The formulation based on Hamilton's equations described in Section 2.3 also benefits from a Newton-Raphson iteration scheme such as the one described in Section 3.2. The adjusted parameters in this case were the penalty factor $\alpha$ and $\xi$, which determines the relative weight of $\boldsymbol{\Phi}$ in Eq. (10). Parameter $\omega$ was set to 1 . The energy drifts obtained in the simulation of the four-bar linkage with the fixed point iteration scheme are shown in Fig. 12. Based on the obtained results, it is difficult to predict whether other pairs of $\alpha$ and $\xi$ will result in a successful simulation. It was observed that the energy drop during the pass through singularities did not remain consistent during some simulations. Significant differences took place as the result of slight variations in the simulation conditions, such as the proximity of the system configuration to the actual singular configuration in the sampled time-steps. Conversely, the Newton-Raphson 


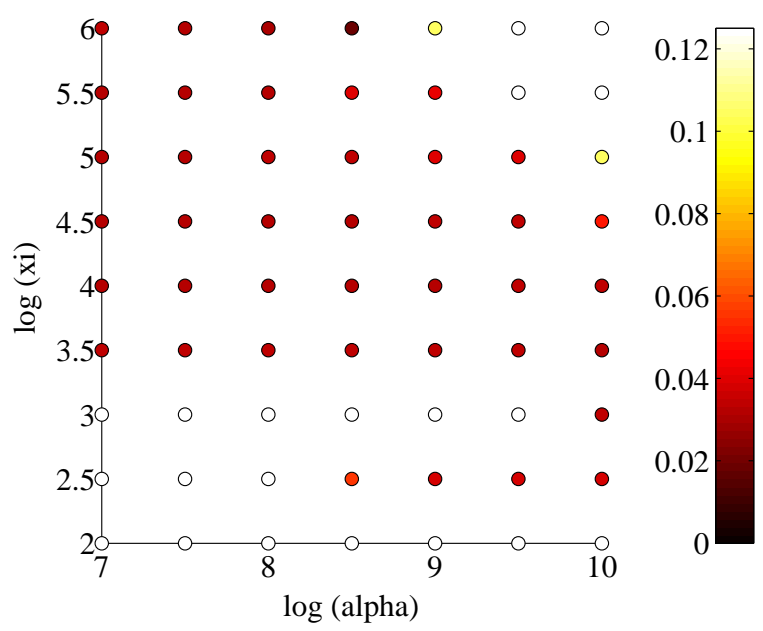

Figure 13: Energy drift during the simulation of the double four-bar linkage, with the augmented Hamiltonian formulation, for different values of the penalty factor $\alpha$ and parameter $\xi$. The trapezoidal rule in Newton-Raphson scheme was used as integrator, with $h=5 \mathrm{~ms}$.

implementation showed a more robust and predictable behaviour. In this case, the valid region is clearly defined, as can be seen in Fig. 13, even though the energy drift is somewhat larger in most cases. The time history of the energy drifts obtained with the two different approaches are compared in Fig. 14 for the case in which $\alpha=10^{8}, \omega=1$, and $h=5$ ms. The fixed point iterative scheme resulted in noticeable energy drops during the pass through some singularities, even in simulations that met the maximum energy drift requirement. Simulations with Newton-Raphson scheme showed much more consistent results for values of $\xi$ within the region of validity in the $\alpha-\xi$ plane.
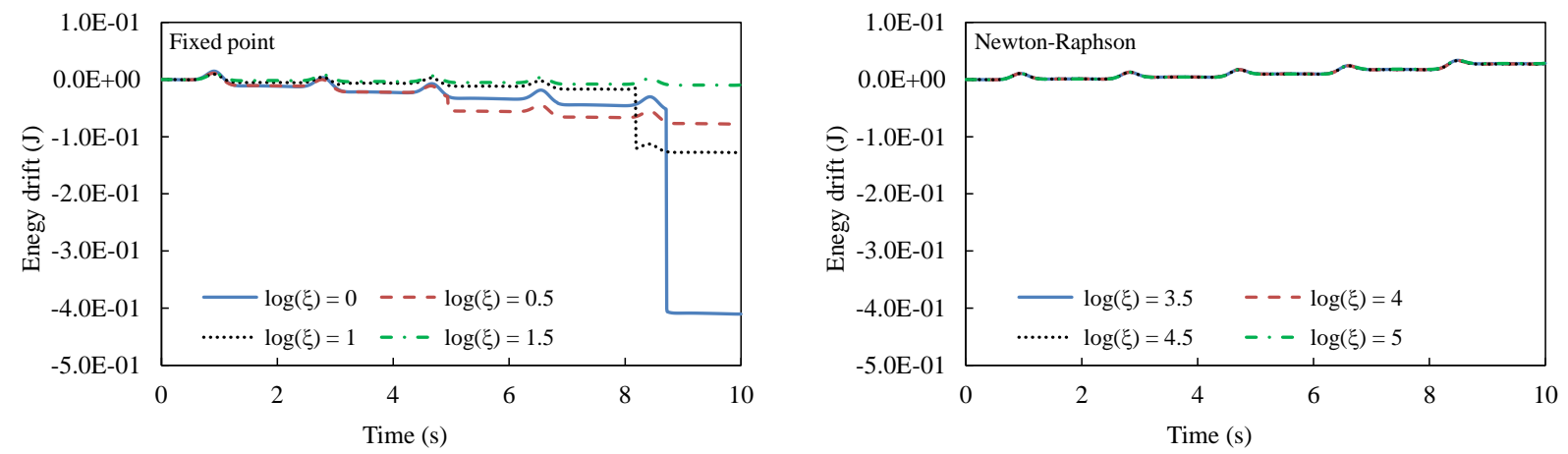

Figure 14: Energy drift of the four-bar linkage during simulation with the augmented Hamiltonian method and the trapezoidal rule $\left(\alpha=10^{8}, \omega=1, h=5 \mathrm{~ms}\right)$ for different values of $\xi$. Results are shown for fixed point (left plot) and Newton-Raphson (right plot) iteration schemes.

Finally, the augmented Lagrangian formulation of index-3 with velocity and acceleration projections (ALi3) featured a remarkably robust performance in the studied examples. The use of mass-orthogonal projections ensured the satisfaction of constraints at the velocity and acceleration levels and removed the need to use Baumgarte stabilization. The penalty factor $\alpha$ thus 
became the only parameter that needed to be tuned up. Simulation results did not change noticeably for a wide range of this parameter. In the case of the four-bar linkage with $h=5 \mathrm{~ms}$, the penalty factor could be varied between $\alpha=10^{6}$ and $\alpha=10^{15}$ without degrading the simulation results. Additionally, this was achieved without a negative impact on efficiency.

\section{Conclusions}

Penalty-based Lagrangian methods for multibody system dynamics can deal with rank deficient Jacobian matrices but still suffer from numerical difficulties near singular configurations. In this research, the source of these problems was determined to be the sudden enlargement of the subspace of admissible motion at the singular points, which introduces impact forces in the constraint reactions. This, in turn, can result in sudden variations of the mechanical energy and eventually cause the simulation to fail. The behaviour of the numerical simulations parallels the one that a physical mechanical system would exhibit in similar conditions: impacts in the joint reactions would be developed in the proximity of singularities due to inaccuracies in manufacturing, clearances, and the deformation of its links.

In this work, benchmark problems were used to compare several augmented Lagrangian formulations in terms of their ability to carry out an efficient simulation while attempting to keep the mechanical energy of the system constant. Implementations of these algorithms in a NewtonRaphson iterative scheme were developed and tested as well. It was found that the selection of the numerical integrator and the tuning of the formulation parameters play a key role in the robustness and accuracy of the simulations. In particular, iterative integrators may diverge at the singularity, and so provisions must be made to stop the iteration process if this happens. In all cases, keeping the constraint violations at the configuration level under a certain threshold was required to obtain a successful simulation. This can constitute a guiding principle in the adjustment of the formulation parameters. Moreover, the formulations must be robust enough to deal with large impact forces. The algorithms in Newton-Raphson form were less sensitive to changes in the formulation parameters, and their efficiency was comparable to their fixed point iteration counterparts in the simulation examples selected in this research. Among these, the augmented Lagrangian formulation of index-3 with velocity and acceleration projections showed the best behaviour in the studied cases. 


\section{Acknowledgements}

The first author would like to acknowledge the support of the Spanish Ministry of Economy through its post-doctoral research program Juan de la Cierva, contract No. JCI-2012-12376.

\section{References}

[1] Baumgarte, J.: Stabilization of constraints and integrals of motion in dynamical systems. Computer Methods in Applied Mechanics and Engineering 1(1), 1-16 (1972). DOI 10. 1016/0045-7825(72)90018-7

[2] Bayo, E., Avello, A.: Singularity-free augmented Lagrangian algorithms for constrained multibody dynamics. Nonlinear Dynamics 5(2), 209-231 (1994). DOI 10.1007/ BF00045677

[3] Bayo, E., García de Jalón, J., Avello, A., Cuadrado, J.: An efficient computational method for real time multibody dynamic simulation in fully Cartesian coordinates. Computer Methods in Applied Mechanics and Engineering 92, 377-395 (1991). DOI 10.1016/ 0045-7825(91)90023-Y

[4] Bayo, E., García de Jalón, J., Serna, M.A.: A modified Lagrangian formulation for the dynamic analysis of constrained mechanical systems. Computer Methods in Applied Mechanics and Engineering 71(2), 183-195 (1988). DOI 10.1016/0045-7825(88)90085-0

[5] Bayo, E., Jiménez, J.M., Serna, M.A., Bastero, J.M.: Penalty based Hamiltonian equations for the dynamic analysis of constrained mechanical systems. Mechanism and Machine Theory 29(5), 725 - 737 (1994). DOI 10.1016/0094-114X(94)90114-7

[6] Bayo, E., Ledesma, R.: Augmented Lagrangian and mass-orthogonal projection methods for constrained multibody dynamics. Nonlinear Dynamics 9(1-2), 113-130 (1996). DOI 10.1007/BF01833296

[7] Blajer, W.: An orthonormal tangent space method for constrained multibody systems. Computer Methods in Applied Mechanics and Engineering 121(1-4), $45-57$ (1995). DOI 10.1016/0045-7825(94)00682-D 
[8] Blajer, W.: A geometrical interpretation and uniform matrix formulation of multibody system dynamics. Zeitschrift fur Angewandte Mathematik und Mechanik 81(4), 247-259 (2001). DOI 10.1002/1521-4001(200104)81:4〈247::AID-ZAMM247〉3.0.CO;2-D

[9] Blajer, W.: Augmented Lagrangian formulation: Geometrical interpretation and application to systems with singularities and redundancy. Multibody System Dynamics 8(2), 141 - 159 (2002). DOI 10.1023/A:1019581227898

[10] Cuadrado, J., Cardenal, J., Bayo, E.: Modeling and solution methods for efficient real-time simulation of multibody dynamics. Multibody System Dynamics 1(3), 259-280 (1997). DOI 10.1023/A:1009754006096

[11] Cuadrado, J., Cardenal, J., Morer, P., Bayo, E.: Intelligent simulation of multibody dynamics: Space-state and descriptor methods in sequential and parallel computing environments. Multibody System Dynamics 4(1), 55-73 (2000). DOI 10.1023/A:1009824327480

[12] Dopico, D., González, F., Cuadrado, J., Kövecses, J.: Determination of holonomic and nonholonomic constraint reactions in an index-3 augmented Lagrangian formulation with velocity and acceleration projections. Journal of Computational and Nonlinear Dynamics 9(4), paper 041,006 (2014). DOI 10.1115/1.4027671

[13] Dopico, D., Luaces, A., González, M., Cuadrado, J.: Dealing with multiple contacts in a human-in-the-loop application. Multibody System Dynamics 25(2), 167-183 (2011). DOI 10.1007/s11044-010-9230-y

[14] Featherstone, R.: A divide-and-conquer articulated-body algorithm for parallel O(log(n)) calculation of rigid-body dynamics. Part 1: Basic algorithm. The International Journal of Robotics Research 18, 867-875 (1999). DOI 10.1177/02783649922066619

[15] Flores, P., Machado, M., Seabra, E., Tavares da Silva, M.: A parametric study on the Baumgarte stabilization method for forward dynamics of constrained multibody systems. Journal of Computational and Nonlinear Dynamics 6(1), paper 011,019 (2011). DOI $10.1115 / 1.4002338$

[16] Goldstein, H.: Classical Mechanics. Addison-Wesley (1980)

[17] González, F., Kövecses, J.: Use of penalty formulations in dynamic simulation and analysis of redundantly constrained multibody systems. Multibody System Dynamics 29(1), 57-76 (2013). DOI 10.1007/s11044-012-9322-y 
[18] González, F., Naya, M.A., Luaces, A., González, M.: On the effect of multirate co-simulation techniques in the efficiency and accuracy of multibody system dynamics. Multibody System Dynamics 25(4), 461 - 483 (2011). DOI 10.1007/s11044-010-9234-7

[19] González, M., Dopico, D., Lugrís, U., Cuadrado, J.: A benchmarking system for MBS simulation software: Problem standardization and performance measurement. Multibody System Dynamics 16(2), 179-190 (2006). DOI 10.1007/s11044-006-9020-8

[20] González, M., González, F., Dopico, D., Luaces, A.: On the effect of linear algebra implementations in real-time multibody system dynamics. Computational Mechanics 41(4), 607-615 (2008). DOI 10.1007/s00466-007-0218-2

[21] IFToMM Technical Committee for Multibody Dynamics: Library of computational benchmark problems (2015). URL http://www . iftomm-multibody.org/benchmark

[22] García de Jalón, J., Bayo, E.: Kinematic and Dynamic Simulation of Multibody Systems. The Real-Time Challenge. Springer-Verlag (1994)

[23] García de Jalón, J., Gutiérrez-López, M.D.: Multibody dynamics with redundant constraints and singular mass matrix: existence, uniqueness, and determination of solutions for accelerations and constraint forces. Multibody System Dynamics 30(3), 311-341 (2013). DOI 10.1007/s11044-013-9358-7

[24] Kövecses, J.: Dynamics of mechanical systems and the generalized free-body diagram part I: General formulation. Journal of Applied Mechanics 75(6), paper 061,012 (2008). DOI 10.1115/1.2965372

[25] Malczyk, P., Fraczek, J.: A divide and conquer algorithm for constrained multibody system dynamics based on augmented Lagrangian method with projections-based error correction. Nonlinear Dynamics 70(1), 871-889 (2012). DOI 10.1007/s11071-012-0503-2

[26] Malczyk, P., Fraczek, J., Chadaj, K.: A parallel algorithm for multi-rigid body system dynamics based on the Hamilton's canonical equations. In: S.S. Kim, J.H. Choi (eds.) Proceedings of the 3rd Joint International Conference on Multibody System Dynamics. Busan, Korea (2014)

[27] Naudet, J., Lefeber, D., Daerden, F., Terze, Z.: Forward dynamics of open-loop multibody mechanisms using an efficient recursive algorithm based on canonical momenta. Multibody System Dynamics 10(1), 45-59 (2003). DOI 10.1023/A:1024509904612 
[28] Newmark, N.M.: A method of computation for structural dynamics. Journal of the Engineering Mechanics Division, ASCE 85(EM3), 67-94 (1959)

[29] Ruzzeh, B., Kövecses, J.: A penalty formulation for dynamics analysis of redundant mechanical systems. Journal of Computational and Nonlinear Dynamics 6(2), paper 021,008 (2011). DOI 10.1115/1.4002510 